\title{
Multiscale Structure of Super Insulation Nano-Fumed Silicas Studied by SAXS, Tomography and Porosimetry
}

Belynda Benane ${ }^{1,2,3}$, Guilhem P. Baeza ${ }^{1,2, *}$, Bruno Chal1, 2, Lucian Roiban ${ }^{1,2}$, Sylvain Meille1, 2, Christian Olagnon¹, 2, Bernard Yrieix², 3 and Geneviève Foray¹, 2

* Corresponding author: guilhem.baeza@insa-lyon.fr, +33 472437258

${ }^{1}$ Univ Lyon, INSA-Lyon, CNRS, UCBL, MATEIS, UMR 5510, 7 avenue Jean Capelle, F-69621, Villeurbanne, France

2 Univ Lyon, INSA-Lyon, CNRS, UCBL, MATEB, 7 avenue Jean Capelle, F-69621, Villeurbanne, France

3 EDF R\&D, Les Renardières, F-77250, Moret sur Loing, France

\begin{abstract}
We focus on describing the multi-scale structure of a fumed silica characterized by remarkably low thermal conductivity (ca. 2-5 $\mathrm{mW} \cdot \mathrm{m}^{-1} \cdot \mathrm{K}^{-1}$ ) when used as a core material in vacuum insulating panels. While such powders are known to be highly polydisperse at different lengthscales (hardly quantifiable), we propose to adapt a recent methodology based on small-angle X-ray scattering experiments with the aim of providing simple criteria for characterizing the morphology of these nanostructured silicas. Combining this technique with transmission electron microscopy, electron-tomography and mercury intrusion porosimetry then allows assigning the origin of the super-insulation to the low dimensionality of the silica aggregates at lengthscales smaller than $500 \mathrm{~nm}$. Remarkably, by using independently these three techniques, we always find the compacity of the aggregates (radius of ca. $40 \mathrm{~nm}$ ) to be equal to $0.29 \pm 0.01$. This study proposes therefore a robust methodology, potentially of a great interest for industrial applications.
\end{abstract}

Keywords: Silica, Insulation, Tomography, Fractal Aggregates, SAXS 


\section{Introduction}

Ranked among multi-scale materials, nanostructured silica powders are composed of nanometer sized elementary particles, organized at higher scales as aggregates and agglomerates.[1] While the former are believed to be indivisible gatherings of elementary particles, the latter consist of clusters of weakly bounded aggregates that can be "broken" by applying mechanical stress, e.g. in nanocomposites, by "internal" mixing [2-4] or in solution via stirring[5] and sonication. $[6,7]$

When loosely packed, these nano-architectured materials are essentially composed of air (>80\%)[8-12] with typical pore sizes not exceeding $200 \mathrm{~nm}$, making them excellent candidates for thermal insulation applications. Indeed, their very open structure (low fractal dimension), combined with the nanometric size of their pores, drastically limits heat conduction through both the solid skeleton and the gas routes.[13-17] Taking advantage of such remarkable properties, a wide range of thermal insulating systems based on nanostructured silicas and aerogels[18-20] have emerged in recent years. Among them, vacuum insulating panels (VIP), made of a compacted silica core in a sealed envelope, have been identified as one of the most promising technologies.[21-23] While the thermal conductivity of conventional thermal insulation materials such as mineral fibers, polyurethane foams and expanded polystyrene is situated between $2510^{-3}$ and $4010^{-3} \mathrm{~W} \cdot \mathrm{m}^{-1} \cdot \mathrm{K}^{-1},[24-26]$ VIPs allow dividing it by as much as a factor of 8 , reaching $2-510^{-3} \mathrm{~W} \cdot \mathrm{m}^{-1} \cdot \mathrm{K}^{-1}$ when their inner pressure is maintained around $100 \mathrm{~Pa}$.[27] They therefore offer major improvements in terms of building renovation, notably by reducing the insulating layer thickness to less than $30 \mathrm{~mm}$ to fulfill current building insulation standards.

Nevertheless, VIP panels are faced by a major barrier to their development and commercialization, namely their price. They remain confined to high added value 
applications, historical renovation, and demonstration sites.[28, 29] In order to enlarge their field of use, many studies have been launched for more than a decade to develop competitive and efficient silica formulations. In particular, two types of nanopowders have been promoted: (i) fumed silicas (FS) synthesized by pyrolysis in a flame up to $1500{ }^{\circ} \mathrm{C}$ and characterized by a hydrophobic surface[30-32] and (ii) precipitated silicas (PS), obtained from aqueous hydrolysis, leading to a quite hydrophilic behavior.[33, 34] The former are currently used in VIPs in spite of their higher cost, because they can be employed to assemble panels with sufficient compression strength for handling at lower density (140 - $200 \mathrm{~kg} \cdot \mathrm{m}^{-3}$, i.e., 6 - 10 vol.\% solid phase). In comparison, the latter exhibit higher densities (200 250 kg.m-3 i.e., 10 - 12 vol.\% solid phase) for a similar compression stress, synonymous with lower insulation properties.[35]

The real challenge therefore resides in identifying the origin of such different behaviors in view to adapting either synthesis routes or composite VIP processing to the industrial scale for the potential mass market. While it is commonly accepted that the macro-properties of a powder are mostly driven by its morphology[36] together with its surface state,[37] a clear picture capable of explaining this structure-property relationship is still missing. As so often in materials science, the task is made difficult by the multi-scale nature of the problem and the lack of a technique able to provide information on the whole range of sizes.

We address this challenge here. In particular, by combining structural characterization in both the direct and Fourier space, we demonstrate that the origin of the high porosity present in fumed silica resides in its pronounced low dimensionality character at the 50-350 nm length scale. On the one hand, transmission electronic microscopy (TEM) and electronic tomography (ET) reveal the 
flat (oblate) or pearl necklace (prolate) shape of the aggregates. On the other hand, in-depth analysis of small angle X-ray scattering (SAXS) patterns allows generalizing this result by suggesting the stacking of elongated objects when the compression stress is increased in powder pellets. Furthermore, we provide a self-consistent model to estimate the volume fraction in aggregates that we subsequently validate using mercury intrusion porosimetry. This original parallel approach opens the way to more systematic structural characterization in this field.

\section{Experimental Section}

Nature of the fumed silica: The fumed silica studied (Konasil 200, OCI Korea) had a skeletal (intrinsic) density of 2.2 g.cm-3, a tapped density close $0.05 \mathrm{~g} . \mathrm{cm}^{-3}$ and a purity higher than 99.8\%.[38] Its typical untapped density is expected to be close to $0.03 \mathrm{~g} . \mathrm{cm}^{-3}$, in fair agreement with the volume fraction of the "powder" estimated from SAXS (Table 1).

Sample preparation: Pellets were shaped by oedometric compression at a 10 $\mathrm{mm} \cdot \mathrm{min}^{-1}$ loading rate, up to either $0.15,0.6$ or $1.2 \mathrm{MPa}$ using a Zwick Roell press equipped with a $500 \mathrm{~N}$ load cell. Sample unloading was performed at $5 \mathrm{~mm} \cdot \mathrm{min}^{-1}$. Pellet masses were always equal to $100 \mathrm{mg}$ to ensure adequate thickness for the SAXS measurements (ca. $2 \mathrm{~mm}$ ). The suspension $(50 \mathrm{~mL}$ ) was prepared by adding the silica powder in ethanol (0.1 vol.\%) and subsequent sonication at 50oW for 1 minute with a Vibra-cell 500 (Sonics, USA) allowing to ensure a good and stable dispersion. During sonication, the vessel containing the suspension was immerged into cold water and ice to limit the ethanol evaporation. The resulting sample was transferred into a 2 mm diameter capillary tube (specialglass, WJ M-Glass) and measured in SAXS ca. 5 minutes later. 
Transmission electron microscopy: TEM analyses were performed on both Jeol 2010F (200 keV) and EFI TITAN environmental TEM (80 keV-300 keV) electron microscopes operating under high vacuum. The samples were prepared using a "dry method" consisting in the following. A small amount of silica (ca. $20 \mathrm{mg}$ ) was first crushed into an agate mortar and transferred into a $25 \mathrm{~cm}^{3}$ closed vessel. The latter was then shook by hand for five minutes in order to generate a "silica fume dust" which was subsequently captured manually with a perforated carbon film cupper grid with a "300" mesh. The images were finally recorded using minimum illumination of the samples to prevent deteriorating the silica.

Electron tomography: Electron tomography[39, 40] consists in recording series of tilted images of an object in order to reconstruct its volume in 3D.[41, 42] In this study, the tilt series were recorded using a EFI TITAN environmental TEM (80 keV$300 \mathrm{keV}$ ). The sample was prepared dry, as explained in the TEM section, using a microscopy grid on which gold nanoparticles of $5 \mathrm{~nm}$ diameter were deposited previously as fiducial markers. The microscopy grid was then set on a Fischione high tilt holder. Because the silica was extremely sensitive to the beam, great care was given to the electron dose received by the sample in order to prevent any damage.[43] 75 images with a pixel size of $0.33 \mathrm{~nm}$ were recorded. Image alignment was performed using the Imod software,[44] the reconstruction was built using 15 iterations of the ART algorithm implemented using TomoJ software and ImageJ.[45] Finally, the data segmentation and quantification were performed by combining tools implemented in ImageJ[46, 47] and 3D Slicer.[48]

SAXS: Ultra-SAXS and SAXS measurements were performed on the beam line IDo2 at the ESRF, Grenoble, France. Two sample-detector distances were used, $1 \mathrm{~m}$ (SAXS) and $10 \mathrm{~m}$ (USAXS) with a beam wavelength of $\lambda=1.1 \AA$ to measure the scattering 
intensity $I(q)$ over three decades of scattering vector $q\left(210^{-3} \mathrm{~nm}^{-1}\right.$ to $\left.2 \mathrm{~nm}^{-1}\right)$, roughly corresponding from $3 \mu \mathrm{m}$ to $3 \mathrm{~nm}$ in real space. The beam was rectangular with an approximate size of $0.1 \times 0.2 \mathrm{~mm}^{2}$. Each diffractogram was obtained by averaging ten measurements performed at different locations in the samples. The few diffractograms presenting singularities (samples heterogeneities) were eliminated. The transmission was systematically found to be in the $0.57-0.68$ range.

Mercury porosimetry: Porosimetry measurements were performed on an AUTOPORE IV 9500 (Micrometrics Instrument Corporation, USA). A powder cell equipped with a $1.131 \mathrm{~cm}^{3}$ capillary and a $5.913 \mathrm{~cm}^{3}$ cell volume was used. All the measurements were corrected with a blank performed on an empty cell. Samples were all $100 \mathrm{mg}$ in mass; a $4 \mathrm{~h}$ void was performed before measurements. The maximum pressure was $200 \mathrm{MPa}$; more than 50 points were recorded during both the loading and unloading sample steps.

\section{Results and Discussion}

\subsection{Direct visualization at the nano-scale}

In Figure 1a we present an electron micrograph of a fumed silica agglomerate of ca. 400-500 $\mathrm{nm}$ in diameter obtained by transmission electron microscopy in bright field mode (BF-TEM). Figures $1 \mathrm{~b}$ and $1 \mathrm{c}$ are images at higher magnifications showing the agglomerate's inner-structure presented in Figure 1a. These micrographs clearly reveal the presence of large pores and denser zones, called aggregates, presenting either flat or necklace shapes at the $100 \mathrm{~nm}$ lengthscale (see e.g. the white ellipsoids in Figures 1e-f). The aggregates are themselves made of silica particles well visible in Figures 1b-c exhibiting elliptical geometries with major and minor radii estimated to 
be $R_{\max }=13-17 \mathrm{~nm}$ and $R_{\min }=5-7 \mathrm{~nm}$, respectively, from image analysis. Careful examination of Figure if also reveals a gradient in terms of particle radius, with the long axes of the particles passing progressively from $6 \mathrm{~nm}$ (bottom left corner) to 17 $\mathrm{nm}$ (top-right corner). This effect has been documented in the literature and is known to be generated by the flame synthesis of fumed silica, causing heterogeneous conditions along the aggregates.[49]

Then, "XY" cross-sections extracted from the reconstructed volume of an agglomerate investigated with electron tomography (Figure 2a-c) confirm the very open nature of such silica powders, regardless of the Z-coordinate. Indeed, removing the volume occupied by the largest pores makes it possible to estimate the compacity of the denser zones within the agglomerates, resulting in ca. $\kappa_{\text {tomo }} \approx 28 \%$. (Details of the data analysis are given in Supplementary Material, Figures SM.1 and SM.2, as well as in the related video SI1_silica_volume.avi). Furthermore, in addition to characterizing the high porosity content, reconstructing the agglomerates in $3 \mathrm{D}$ (Figures 2d-f) allows selectively scanning sub-parts of the objects, corroborating the elongated character of the aggregates at the $100 \mathrm{~nm}$ lengthscale. Indeed, Figures 2e and $2 \mathrm{f}$ show the presence of flat and necklace-shaped aggregates, respectively, confirming the previous TEM observations.

Based on these initial investigations, it appears possible to rationalize from simple geometrical arguments the lower apparent density of the compacts of fumed silica in comparison with their precipitated counterparts (mostly made of "more spherical" aggregates[1, 43]). In order to support and deepen the above investigation performed on limited pieces of materials, we propose in the following section to use a systematic SAXS description of the fumed silica prepared from different conditions. 


\subsection{Small Angle X-ray Scattering}

In Figure 3a, we present the scattering intensity $I(q)$ measured from different samples made of fumed silica: (i) three pressed pellets prepared by applying 0.15, o.6 and 1.2 MPa in an oedometer cell (20 mm of diameter), (ii) the corresponding unpressed powder, and (iii) a suspension in ethanol with a nominal volume fraction in silica of 0.1 vol.\%. As expected, all the samples made of silica in air ((i) and (ii)) exhibit similar intensities at high- $q$, while the silica in ethanol scatters much less due to a weaker electronic contrast between the two phases. Once normalized by the volume fraction of silica $\Phi_{s i}$, i.e., the ratio of the volume truly occupied by the solid matter over the volume of the sample, calculated from the pellet's dimension of ca. $650 \mathrm{~mm}^{3}$ and its mass, the pellets' signals all collapsed above $1 \mathrm{O}^{-1} \mathrm{~nm}^{-1}$. This allowed extracting subsequently the volume fraction of silica within the unpressed powder ( $\approx 11$ vol.\%) and the corresponding "true" value for the suspension ( $\approx 0.07$ vol.\%). Note that in the latter case, the signal had to be multiplied beforehand by the contrast ratio $\Delta \rho_{\text {SiO2-air }}^{2} / \Delta \rho_{\text {SiO2-EtoH }}^{2}$, with $\Delta \rho_{i-j}^{2}$ being the SAXS contrast in a biphasic (i, j) medium[50] (see Supplementary Material - Notes). These results are grouped in Figure $3 \mathrm{~b}$ and must be read in the following way:

For $\mathbf{q}>\mathbf{0 . 3} \mathbf{n m}^{-1}$ : This $q$-range (momentum transfer), known as the Porod regime,[50] is indicative of the smallest objects within the material only, i.e., the elementary nanoparticles. Once the data are properly normalized (by $\Phi_{s i}$ and $\Delta \rho_{i-j}^{2}$ in $\mathrm{cm}^{-4}$ ), they all overlap regardless of the structure at higher lengthscales. The assumption of perfect sphericity allowed estimating the radius of these "buildingblocks" through a simple power-law fit of the data such that $I(q)=A q^{-4}$ (see Figure 3a-b). Here, exponent 4 stands for a perfectly smooth interface, in quite good 
agreement with Figure 1, while pre-factor $A$ can be used to calculate the Porod radius through the following expression:

$$
R_{p}=\frac{6 \pi \Phi_{s i} \Delta \rho_{S i O 2-a i r}^{2}}{A}
$$

Based on the estimation of $\Delta \rho_{\text {Sion-air }}^{2}$, (see Supplementary Material - Notes), we obtain $R_{p} \approx 7.0 \mathrm{~nm}$, a characteristic size resulting in a nanobead volume of $V_{p}=$ $\frac{4}{3} \pi R_{p}^{3}=1.410^{3} \mathrm{~nm}^{3}$, in comparison with the ellipsoid geometry defined in the previous section $V_{\text {ellipsoid }}=\frac{4}{3} \pi R_{\max } R_{\min }^{2}=2.210^{3} \mathrm{~nm}^{3}$. A perfect match would have been obtained for $R_{p}=8.1 \mathrm{~nm}$, giving an idea of the error bar.

For $0.1>$ q $>0.3 \mathbf{~ n m}^{-1}$ : This short transition zone presents a change in slope, corresponding to the inflexion point of the nanoparticle form factor. The latter is usually described from the Guinier approximation used to extract the radius of gyration $R_{g}$ of the scattering objects with the expression $I(q)=I_{0} \exp \left(-\frac{q^{2} R_{g}^{2}}{3}\right)$, where $I_{0}=V_{p} \Phi_{s i} \Delta \rho_{\text {SiO2-air }}^{2}$. Note that in Figure $3 \mathrm{~b}$, a calculation based on the silica-air Xray contrast $\left(\Delta \rho_{\text {SiO2-air }}^{2}\right)$ and the Porod radius $\left(R_{p}\right)$ with the empirical Guinier-Porod model[51] (assuming $R_{g}=R_{p}$ ), perfectly matches the experimental data, highlighting the self-consistency of the two descriptions.

For $\mathbf{q}<\mathbf{0 . 1} \mathbf{n m}^{-1}$ : This $q$-range concerns the powder structure at higher lengthscales, namely the aggregates and agglomerates - spanning almost two decades in direct space from $\approx 50 \mathrm{~nm}$ to $2 \mu \mathrm{m}$ (see Figures 1 and 2 ). Immediately below $q=0.1 \mathrm{~nm}^{-1}$, the different signals start to separate, with the denser systems exhibiting lower intensity. This trend indicates the presence of a deeper structure factor "correlation hole" in more compressed pellets rather than the decreasing size of the aggregates (related to their form factor). $[1,52]$ To eliminate the structure factor from the measurement, we 
thus measured a very diluted and sonicated silica suspension ( $\approx 0.07 \mathrm{vol} . \%)$ in ethanol providing us with the apparent form factor of the agglomerates $I_{0} P_{\text {agglo }}(q)$ (grey signal called "Suspension" in Figure 3a-c; see also the schematic representation in Figure 5a). As can be seen, the derivative of that signal with respect to $q$ is monotonic - a strong argument suggesting, in fact, that most of the contribution of the interaggregate structure factor has been eliminated.[53]

This important data then allows extracting the most probable gyration radius of the aggregates and the agglomerates from the breaks in slope positions observed in Figure $3 \mathrm{~b}$, respectively $R_{\text {agg }}=\pi / q^{*}$ and $R_{\text {agglo }}=\pi / q^{* *}$. Alternatively, one can use the Kratky representation (i.e. $\left.I(q) q^{2}=f(q)\right)[1,54]$ to make emerge more clearly these two characteristic sizes through the formation of a trapeze-shaped signal in which the power law and the breaks in slope in Figure $3 \mathrm{~b}$ are transformed respectively into a plateau and its two extremities in Figure 3c. Based on the latter, we estimated the following values: $q^{*}=810^{-2} \pm 110^{-2} \mathrm{~nm}^{-1}$ and $q^{* *}=910^{-3} \pm 110^{-3} \mathrm{~nm}^{-1}$, leading to $R_{\text {agg }}=40 \pm 5 \mathrm{~nm}$ and $R_{\text {agglo }}=350 \pm 50 \mathrm{~nm}$, in agreement with the micrographs presented in Figure 1. Note that such a plateau appears only if the fractal dimension of the agglomerates, readable from $D_{\text {agglo }}^{f}=\left.\frac{d \log \left(I\left(q<q^{*}\right)\right)}{d \log (q)}\right|_{\Phi_{S i} \rightarrow 0} \approx 1.8$ (i.e., the slope of $I(q)$ between $q^{*}$ and $q^{* *}$ in a $\log$-log representation), is close to the $q$ exponent used for the Kratky plot (set as 2 in the present work).

Here, we want to insist on the fact that the fractal dimension of the agglomerates should be extracted only from such "structure factor free" signals ("Suspension" in Figure 3), contrary to what is frequently proposed in the literature.[4, 55, 56] In such cases, the scattering intensity at intermediate $q\left(10^{-2}-10^{-1} \mathrm{~nm}^{-1}\right.$, i.e., $\left.q^{* *}<q<q^{*}\right)$ originates from the product of the aggregate form factor and the inter-aggregate 
structure factor, leading to the erroneous quantification of the fractal dimension of bigger objects when simply taken as the slope of the (log-log) signal. We believe that this confusion comes from the absence of a clear maximum on $I(q)$ and that the authors would not have performed such an analysis in the presence of well-defined structure peaks, like e.g., in colloidal systems. In comparison, this method would have led in our case to $D_{\text {agglo }}^{f}$ varying from 1.0 to 2.0 for the 1.2 MPa pellet and the suspension in ethanol, respectively. In other words, it would have suggested that the density (or compacity) of the agglomerates is much higher in a sonicated suspension than in a pellet formed under high compression.

In addition, the utilization of the Kratky representation to transform the inflexion points of the multiscale form factor into a "trapezoid" or "peak" signal, presents the same restrictions. As can be seen clearly in Figure 3c, the Kratky peak position dramatically shifts to higher $q$, passing from the suspension to the unpressed powder and pellets. Here again, this is unambiguously due to the correlation hole of the interaggregate structure factor, which pulls the signal down at low- $q$ for high $\Phi_{s i}$ values, and certainly not to a dramatic decrease of the aggregate radius. (Indeed, we may have expected the opposite trend, i.e., smaller aggregates in the dispersed state).

Returning to the analysis of the fumed silica, measuring $I_{0} P_{\text {agglo }}(q)$ also makes it possible to calculate the apparent structure factor $S_{a p p}(q)$ in the unpressed powder and pellets (Figure 4a) such that:

$$
S_{a p p}(q)=\frac{I(q)}{I_{0} P_{a g g l o}(q)}
$$

As expected, all the apparent structure factors merge at high- $q$ (same elementary particles) and show a clear correlation hole (destructive interferences) from $0.1 \mathrm{~nm}^{-1}$ 
and below. Interestingly, no major constructive interferences (e.g. structure peaks) appear in these functions, in line with what was observed by Baeza et al.[1,53] and Genix et al.[52] for similar powders but in contrast with experiments performed on colloidal silicas.[57-59] This qualitative difference is usually explained by the considerable polydispersity in both the size $(\sigma>0.3)$ and shape of the fumed/precipitated powders, as shown by simulations.[52] On a more quantitative level, the minimum of $S_{a p p}(q)$, which is related to the isothermal compressibility[50] and denoted $S_{\min }$ hereafter, was used to estimate the volume fraction in silica aggregates within nanocomposites through simulations and the approximation of Percus-Yevick (PY).[60] In our case, the $S_{\min }$ values can be obtained directly through the experimental determination of $P_{a g g l o}(q)$ (Figure 4a) to estimate the volume fraction in aggregates $\Phi_{\text {agg }}$ (see Figure 4b) as shown in equation 3. (Note that $\Phi_{\text {agg }}$ is here formally defined as the ratio of the volume occupied by spheres of radius $R_{a g g}$ containing both silica and air over the volume of the whole sample).

$$
S_{\min } \equiv S_{P Y}(q \rightarrow 0)=\frac{\left(1-\alpha \Phi_{a g g}\right)^{4}}{\left(1+2 \alpha \Phi_{a g g}\right)^{2}}
$$

This function was calculated analytically for monodisperse spheres with no interactions,[60, 61] and recently extended empirically for polydisperse spheres with moderate attraction.[1] Here, $\alpha$ is related to the polydispersity (standard deviation) $\sigma$ by $\alpha=1-1.13 \sigma^{2}$ as defined in ref.[52] for an infinite assembly of particles.

By using a log-normal distribution and $\sigma=0.5$, i.e. a geometrical standard deviation $G S D=\exp (\sigma)$ close to 1.65 as estimated from refs,[49], [62] it is therefore possible to estimate the volume fraction in aggregates for the different samples. We find respectively $\Phi_{\text {agg }}=0.17,0.28,0.35$ and 0.40 for the unpressed powder, and the 
pellets pressed at $0.15,0.6$ and 1.2 MPa. From these values, we can then determine the compacity of the aggregates, i.e., their volume fraction in silica particles through $\kappa_{S A X S}=\Phi_{s i} / \Phi_{a g g}$. Then, one can eventually calculate their corresponding fractal dimensions through $D_{\text {agg }}^{f}=\frac{\ln \left(\kappa_{S A X S}\left(R_{a g g} / R_{p}\right)^{3}\right)-\ln \left(k_{0}\right)}{\ln \left(R_{a g g} / R_{p}\right)}$ but has to keep in mind that such a definition is formally restricted to the case of non-interpenetrated objects only, i.e., applicable, at most, to the uncompressed powder in our work (see Figure $5 \mathrm{~b}$ ). The latter equation is derived from the expression of the aggregation number, i.e., the most probable number of elementary beads contained in an aggregate $: N_{\text {agg }}=$ $k_{0}\left(\frac{R_{a g g}}{R_{p}}\right)^{D_{a g g}^{f}}$, where $k_{0}$ is called the "fractal prefactor" or lacunarity, [49] likely to be related with the degree of overlap between the elementary beads. While its value is often taken as 1 in the literature, $[1,56]$ Oh and Sorensen showed that higher values were more sensible for DLCA morphologies such as fumed silica.[63] In consequence, we fixed $k_{0}=1.3$ in our analysis. The corresponding outputs $\left(\Phi_{a g g}, \kappa_{S A X S}\right.$ and $D_{\text {agg }}^{f}$ ) are regrouped in Table 1 with the rest of the samples characteristics. For the sake of comparison, we also report apparent value of $D_{a g g}^{f}$ for the pellets in the legend $\left(^{*}\right)$ under the symbol $D_{a g g}^{f-a p p}$.

Importantly, while in the suspension the appellation "aggregate" is referring to noninterpenetrated sub-domains of isolated agglomerates, it rather refers to spheres of radius $R_{a g g}$ in which the latter objects become interpenetrated, resulting in a higher apparent compacity in the untapped powder and the pellets (Figure 5). Also, it is worth to note that the calculation of the aggregates compacity $\kappa_{S A X S}$ is independent of any object size. On the contrary, its transformation into an (apparent) aggregate 
fractal dimension $D_{\text {agg }}^{f}\left(\right.$ or $D_{\text {agg }}^{f-a p p}$ ) is calculated from $R_{p}=7.0 \mathrm{~nm}$ and $R_{\text {agg }}=40 \mathrm{~nm}$ (see above), assuming an identical compacity for all the aggregates.

Table 1: Structural characteristics of the fumed powder from the SAXS analysis.

\begin{tabular}{ccccccc}
\hline Sample & $\Phi_{S i}{ }^{(0)}$ & $S_{\min }{ }^{(0)}$ & $\Phi_{\text {agg }}{ }^{(1)}$ & $\kappa_{S A X S}{ }^{(1)}$ & $D_{\text {agg }}^{f}{ }^{(2)}$ & $N_{\text {agg }}{ }^{(2)}$ \\
& \pm 0.01 & \pm 0.02 & \pm 0.05 & \pm 0.1 & \pm 0.05 & \pm 30 \\
\hline Powder & 0.02 & 0.38 & 0.17 & 0.18 & 1.85 & 33 \\
$0.15 \mathrm{MPa}$ & 0.08 & 0.20 & 0.28 & 0.29 & $*$ & 53 \\
$0.6 \mathrm{MPa}$ & 0.10 & 0.14 & 0.35 & 0.29 & $*$ & 53 \\
$1.2 \mathrm{MPa}$ & 0.11 & 0.10 & 0.40 & 0.28 & $*$ & 51 \\
\hline
\end{tabular}

(о) $\Phi_{s i}$ and $S_{\min }$ are measured experimentally by using SAXS.

${ }^{(1)} \Phi_{\text {agg }}$ and $\kappa_{S A X S}$ are calculated from the SAXS measurements assuming $\sigma=0.5$

(2) $D_{a g g}^{f}$ and $N_{a g g}$ are calcuculated from $\kappa_{S A X S}$ with $R_{a g g}=40 \mathrm{~nm}, R_{p}=7.0 \mathrm{~nm}$ and $k_{0}=1.3$.

(*) $D_{\text {agg }}^{f-a p p}=2.13,2.13$ and 2.11 for the pellets pressed at $0.15,0.6$ and $1.2 \mathrm{MPa}$ respectively.

This approach allows highlighting the iso-structure of the aggregates in all the pellets regardless of the pressure applied in the 0.15 MPa - 1.2 MPa range. As a matter of fact, they systematically appear to have a compacity around $\kappa_{S A X S}=0.29$, confirming that the silica structure is not impacted significantly by the compressive stress from the aggregate lengthscale and below. This trend, as well as the values of $D_{a g g}^{f}$ (and eventually $D_{a g g}^{f-a p p}$ ), are found to be in good/fair agreement with other works.[1, 49, $64,65]$ Interestingly, in the untapped powder, in which the interpenetration of the agglomerates is presumably modest, the fractal dimension of the aggregates (seen as spheres of radius $R_{a g g}$ ) is found to be $D_{a g g}^{f} \approx 1.85$, close to the fractal dimension of the agglomerates observed in the suspension $\left(D_{\text {agglo }}^{f} \approx 1.80\right.$ ). This result suggests 
therefore that "native" aggregates and agglomerates could be seen as self-similar objects, as one could have hypothesized from the almost constant exponent of the $I(q)$ power law up to $\pi / R_{p} \mathrm{~nm}^{-1}$ in Figure $3 \mathrm{a}-\mathrm{b}$. Although this result is supported by Boldridge ( $D_{\text {agg }}^{f} \approx 1.86$ ),[49] we want to point here that higher values, closer to $D_{\text {agg }}^{f} \approx 2.31$, could have been obtained by taking $\sigma$ and $k_{0}$ respectively to 0.3 and 1.0 as proposed by Baeza et al.[1] This would have meant that the compacity of the structure tend to decrease significantly with increasing the lengthscale, a sensible result often discussed in the literature.

To summarize our SAXS analysis we refer the reader to the Figure 5. First, one should consider the situation in the suspension (Figure 5a) where silica agglomerates are well separated, giving the opportunity to measure their form factor since at low volume fraction in silica ( $\Phi_{s i} \approx 0.1$ vol.\%) one obtains $I(q)=I_{0} P_{\text {agglo }}(q)$. In this case, the aggregates interact with their neighbors which belong to the same agglomerate only. The fractal dimension of the agglomerates can be then extracted from the power law between $q^{* *}$ and $q^{*}$, i.e. $D_{\text {agglo }}^{f}=1.80$. The corresponding value for the "native" aggregates can be roughly extrapolated to the same value through our model by considering a high polydispersity of the aggregates $(\sigma=0.5)$ and a lacunarity higher than $1\left(k_{0}=1.3\right)$.

Passing then to the untapped powder (Figure $5 \mathrm{~b}$ ) is synonymous of increasing substantially the volume fraction of silica ( $\Phi_{s i} \approx 3$ vol.\%). In this situation, the agglomerates are in contact and are likely to be interpenetrated considering their very open structure. This results therefore in additional interactions between the aggregates, making emerge the inter-aggregates structure factor which pulls down the 
scattering intensity at $<q^{*}$. From this stage, a sphere of radius $R_{a g g}$ is thus containing more silica than in the previous case, making in consequence the apparent fractal dimension of the aggregates slightly higher $D_{a g g}^{f}=1.85$. (That extra-silica comes either from different interpenetrated agglomerates or from the same one, once compressed).

Finally, the compression of the powder into pellets is leading to an even denser material in which the agglomerates are more interpenetrated (Figure 5c). In this context, the aggregates are in close contact with plenty others, particularly if they were originally elongated, resulting in a much lower isothermal compressibility quantifiable in SAXS through the fall of $S_{\min }$ (Figure 4). Following this logic, one can therefore rationalize the results reported in the Table 1 , notably the significant raise of $\kappa_{S A X S}$ from 0.18 to 0.28 when passing from the powder to the compressed pellets. Beyond the strong impact of the elongated nature of the aggregates on the structure of densely packed samples, it is also playing a crucial role on their mechanical properties. As a matter of fact, the fumed silica made of such low-dimensionality objects was found to have a lower percolation threshold in comparison with its precipitated counterpart, leading to enhanced mechanical properties at higher porosity content.[66-68] (The systematic comparison of both FS and PS mechanical properties will be dealt with in our next article).

\subsection{Porosimetry}

In order to compare the above results with macroscopic measurements, we performed mercury porosimetry experiments in a similar way as what was proposed by Smith et al.,[69] to extract the cumulated pores volume contained in the powder at different lengthscales.[70, 71] For fumed silica, the fraction in macropores (in this 
case corresponding to inter-aggregate pores) was measured as they buckled or bent under low mercury pressure. Also, the fraction in mesopores (in this case corresponding to intra-aggregate pores) was measured as mercury infiltrated the material.[72] In Figure 6, we present the typical relative pore volume content vs. pressure curves which clearly illustrate this duality, with the macro- and meso-pores fraction linked to the pinkish and blue zones, respectively. From the quantitative angle, these results show that in a pellet containing $\Phi_{s i}=11$ vol.\% (pressed at 1.2 $\mathrm{MPa}$ ), $71 \%$ of the pores are identified as "macro" while only $29 \%$ are identified as "meso" (see the orange and blue arrows in Figure 6 for a schematic representation of these two pores' categories). For further details on less compacted pellets with a higher macropores content, we refer the reader to the Supplementary Material Figure SM.3 and Notes.

Therefore, by taking into account the solid fraction in the sample $\left(\Phi_{s i}\right)$, we obtain in a straightforward way the volume fraction in the macro- and meso-pores within the pellet, i.e. $P_{\text {macro }}=63$ vol. $\%$ and $P_{\text {meso }}=26$ vol.\%, respectively. Remarkably, adding the former value to the volume fraction of aggregates obtained from the above SAXS analysis $\left(\Phi_{\text {agg }}=40 \mathrm{vol} . \%\right)$, results in $P_{\text {macro }}+\Phi_{a g g} \approx 1$, providing therefore the quantitative confirmation that the material is composed of hollow aggregates and macropores when observed at a lengthscale larger than $100 \mathrm{~nm}$.

At a smaller lengthscale, considering the mesopores as intra-aggregate cavities allows calculating the compacity of the aggregates from the porosimetry outputs such that $\kappa_{\text {poro }}=\frac{V_{s i-i n-a g g}}{V_{a g g}}=\frac{V_{s i-i n-a g g}}{V_{s i-i n-a g g}+V_{\text {meso }}}$, where $V_{\text {si-in-agg }}$ and $V_{\text {meso }}$ are the volume of silica and mesopores, respectively, within an aggregate of volume $V_{a g g}$. From this simple definition, we can then describe the whole sample by multiplying both the numerator 
and the denominator by $\frac{N}{V_{t o t}}(N$ is the number of aggregates in the pellet of volume $\left.V_{\text {tot }}\right)$, resulting in $\kappa_{\text {poro }}=\frac{N V_{s i-i n-a g g} / V_{\text {tot }}}{N\left(V_{s i-i n-a g g}+V_{\text {meso }}\right) / V_{\text {tot }}}$ or more simply, $\kappa_{\text {poro }}=\frac{\Phi_{s i}}{\Phi_{s i}+P_{\text {meso }}}$. The result of this calculation, leading to $\kappa_{\text {poro }}=0.30$, is again in excellent agreement with the SAXS predictions regarding aggregates compacity $\left(\kappa_{S A X S}=0.28\right.$ in Table 1$)$ and further supports the initial tomography estimations, i.e., $\kappa_{\text {tomo }} \approx 0.28$ ), highlighting the robustness of our study. As a matter of fact, the connections between these three parameters obtained from experiments probing the material at different lengthscales represents, in our opinion, a significant progress in terms of structural description. (The aggregate compacity obtained from tomography, SAXS and porosimetry, is actually "averaged" in the present work at the $100 \mathrm{~nm}, 1 \mathrm{~mm}$, and $1 \mathrm{~cm}$ lengthscales respectively).

\section{Conclusion}

To summarize, we used a complementary set of experimental techniques in both direct and Fourier spaces to fully quantify the multiscale structure (5-500 nm) of an industrially relevant fumed silica. We showed in particular that its high porosity, responsible for its outstanding thermal insulation properties, originated from the anisotropic nature of its constituents at the sub-micronic lengthscale. Since the latter characteristic was undoubtedly attributable to the synthesis route, it therefore appears to be of prime importance for the development of innovative powders with reduced costs and higher energetic efficiency.

From a more fundamental point of view, we adapted in this work a clear methodology for the treatment of complex SAXS data measured on highly polydisperse systems. In particular, this approach offers the possibility of extracting basic geometrical 
parameters such as elementary particle radius, mean aggregates size and compacity, all of which being highly relevant as inputs for computer simulations. Last but not least, and for the first time to our knowledge, we were able to establish a strong correlation between SAXS and mercury porosimetry experiments at two different lengthscales (intra- and inter-aggregates). 
Table 2: Parameters and variables used in the article.

\begin{tabular}{|c|c|c|}
\hline Full Name & Symbol & unit \\
\hline \multicolumn{3}{|l|}{ SAXS (experiments and model) } \\
\hline X-ray beam wavelength & $\lambda$ & $\AA$ \\
\hline Scattering vector (Momentum Transfer) & $q$ & $\mathrm{~nm}^{-1}$ \\
\hline Characteristic wave vector related with the aggregates size & $q^{*}$ & $\mathrm{~nm}^{-1}$ \\
\hline Characteristic wave vector related with the agglomerate size & $q^{* *}$ & $\mathrm{~nm}^{-1}$ \\
\hline Scattering intensity & $I(q)$ & $\mathrm{cm}^{-1}$ \\
\hline Scattering intensity prefactor & & $\mathrm{cm}^{-1}$ \\
\hline SAXS contrast between silica and air & $\Delta \rho_{\text {SiO2-air }}^{2}$ & $\mathrm{~cm}^{-4}$ \\
\hline SAXS contrast between silica and ethanol & $\Delta \rho_{\text {SiO2-EtOH }}^{2}$ & $\mathrm{~cm}^{-4}$ \\
\hline SAXS contrast between generic $i$ and $j$ species & $\Delta \rho_{i-j}^{2}$ & $\mathrm{~cm}^{-4}$ \\
\hline Prefactor of the Porod law & & $\mathrm{cm}^{-1} \mathrm{~nm}^{-4}$ \\
\hline Agglomerate form factor & $P_{\text {agglo }}(q)$ & - \\
\hline Apparent structure factor & $S_{a p p}(q)$ & - \\
\hline Minimal value of the apparent structure factor & $S_{\min }$ & - \\
\hline Theoretical structure factor based on the Percus-Yevick approximation & $S_{P Y}(q)$ & - \\
\hline Standard deviation on the aggregate radius (log-normal distribution) & & - \\
\hline Empirical parameter related with the aggregate radius distribution & $\alpha$ & - \\
\hline \multicolumn{3}{|l|}{ Geometrical parameters } \\
\hline Radius of the elementary bead (Porod model) & $R_{p}$ & $\mathrm{~nm}$ \\
\hline Radius of gyration the elementary bead (Guinier model) & $R_{g}$ & $\mathrm{~nm}$ \\
\hline Small radius of the elementary bead (ellipsoid geometry) & $R_{\min }$ & $\mathrm{nm}$ \\
\hline Large radius of the elementary bead (ellipsoid geometry) & $R_{\max }$ & $\mathrm{nm}$ \\
\hline Most probable radius of gyration of an aggregate & $R_{a g g}$ & $\mathrm{~nm}$ \\
\hline Volume associated with the elementary bead (Porod model) & $V_{p}$ & $\mathrm{~nm}^{3}$ \\
\hline Volume associated with the elementary bead (ellipsoid geometry) & $V_{\text {ellispoid }}$ & $\mathrm{nm}^{3}$ \\
\hline Volume associated with the most probable aggregate radius & $V_{a g g}$ & $\mathrm{~nm}^{3}$ \\
\hline Volume occupied by the silica beads in an aggregate & $V_{\text {si-in-agg }}$ & $\mathrm{nm}^{3}$ \\
\hline Volume occupied by the mesopores in an aggregate & $V_{\text {meso }}$ & $\mathrm{nm}^{3}$ \\
\hline Total volume of the sample & $V_{\text {tot }}$ & $\mathrm{nm}^{3}$ \\
\hline Number of aggregates in the whole sample & $N$ & - \\
\hline Volume fraction in silica in the sample & $\Phi_{s i}$ & - \\
\hline Volume fraction in aggregates in the sample & $\Phi_{a g g}$ & - \\
\hline Compacity of the aggregates estimated from tomography & $\kappa_{\text {tomo }}$ & - \\
\hline Compacity of the aggregates estimated from SAXS & $\kappa_{\text {saxs }}$ & - \\
\hline Compacity of the aggregates estimated from porosimetry & $\kappa_{\text {poro }}$ & - \\
\hline Average fraction of macropores in the sample & $P_{\text {macro }}$ & - \\
\hline Average fraction of mesopores in an aggregate & $P_{\text {meso }}$ & - \\
\hline Volume fractal dimension of the aggregates (powder) & $D_{a g g}^{f}$ & - \\
\hline Apparent volume fractal dimension of the aggregates (pellets) & $D_{a g g}^{f-a p p}$ & - \\
\hline Volume fractal dimension of the agglomerates & $D_{\text {agglo }}^{f}$ & - \\
\hline
\end{tabular}




\section{Acknowledgements}

All the authors are grateful to Michael Sztucki (ESRF Grenoble) for his technical support with the SAXS experiments and to CLYM for access to the microscopes. They also gratefully acknowledge funding from CIFRE grant 2014-0903, ADEME grant 1604Co019, and MATeB (joint MATEIS EDF R\&D laboratory). All the authors are sincerely grateful to the Acta Materialia reviewers for their great contributions to this article.

\section{Supplementary Material}

Supplementary Material is available from the Acta Materialia website or from the author.

\section{Conflict of Interest}

The authors declare no conflict of interest 


\section{Figures}
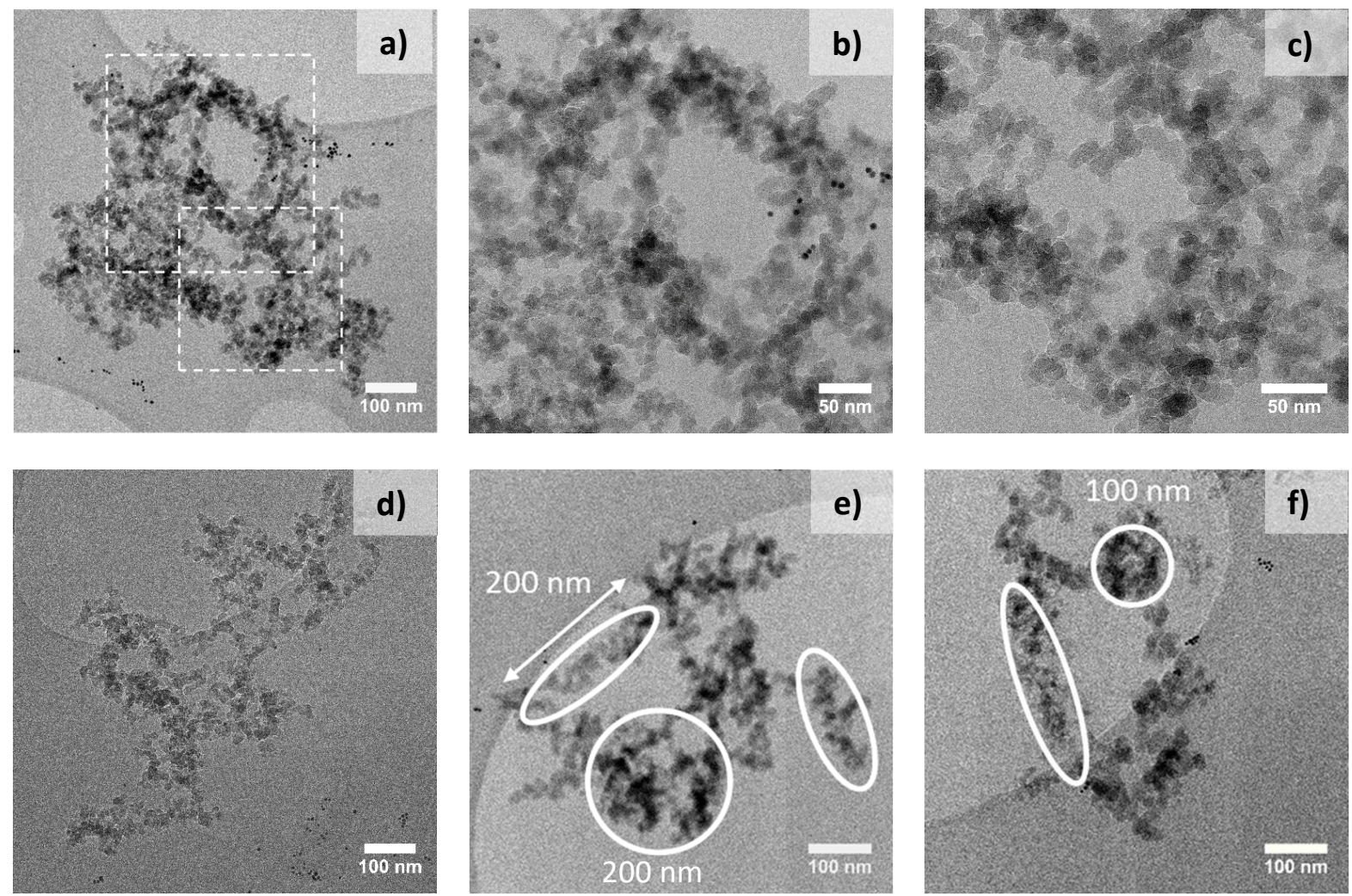

Figure 1: TEM micrographs of the fumed silica. a) a view of a silica agglomerate showing the net structure at the nanoscale. The white dashed rectangles stand for the areas displayed at a higher magnification in b-c) highlighting the internal aggregate morphologies. Necklace assemblies of particles with bimodal porosity stand out. d-e) The aggregates have different morphologies in which "linear" and "flat" local organizations are combined with irregular rough walls one to four particles thick. f) An aggregate formed by silica particles presenting a gradient of radii ranging from 6 $\mathrm{nm}$ to $12 \mathrm{~nm}$ recalls the temperature gradient occurring during the flame synthesis. 

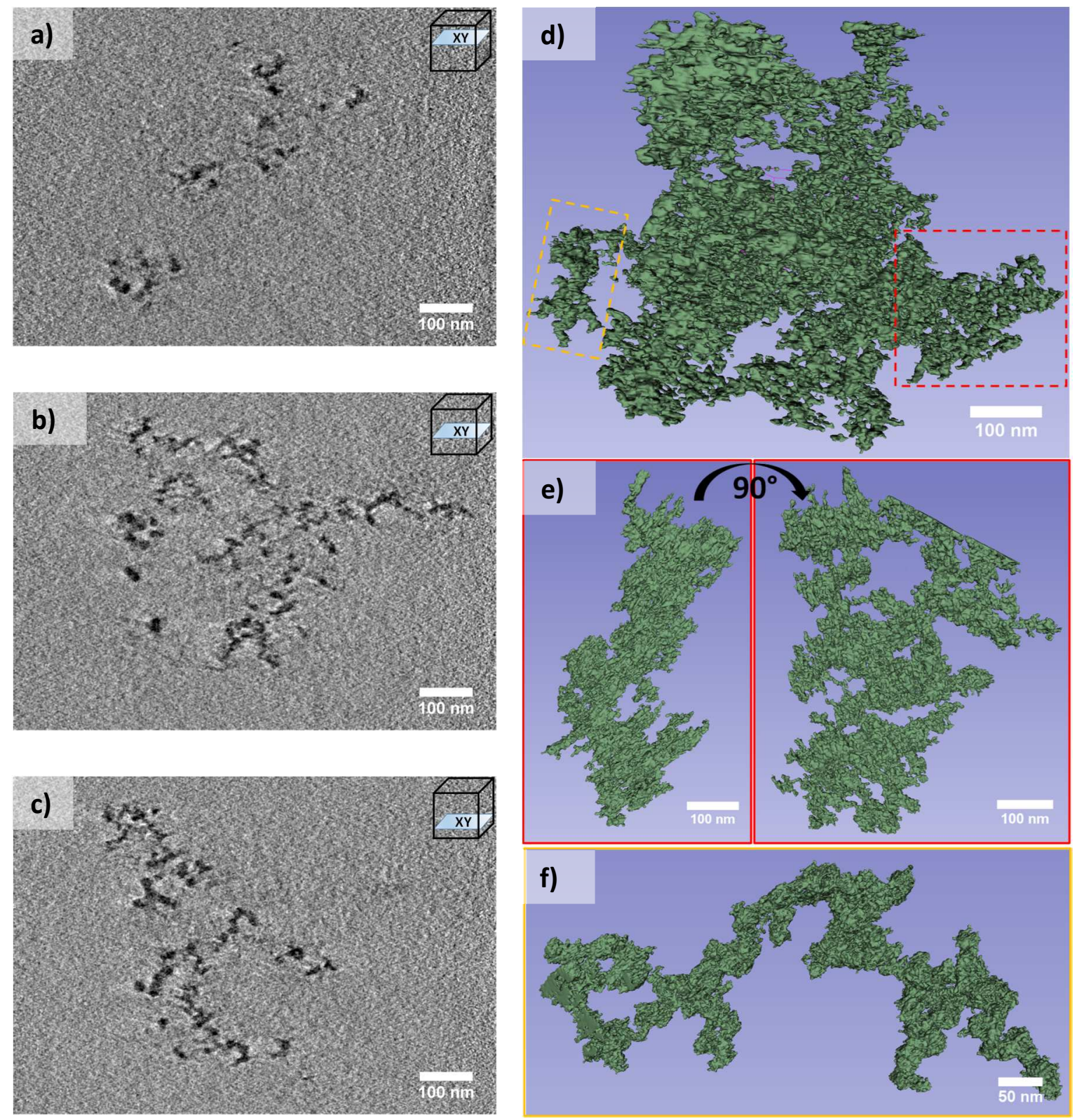

Figure 2: Electron tomography of an agglomerate of fumed silica. a-c) XY cross sections extracted from the reconstructed volume of a silica agglomerate at different depths. The darker gray level represents the silica particles. d) $3 \mathrm{D}$ reconstruction of the whole agglomerate. e) Part of the agglomerate corresponding to the red dashed rectangle in d). The model is displayed in two perpendicular views, revealing the assembly of silica particles in a "flat" shape. f) Part of the agglomerate corresponding to the orange dashed rectangle in d), showing a necklace-like assembly of silica particles. 
a)

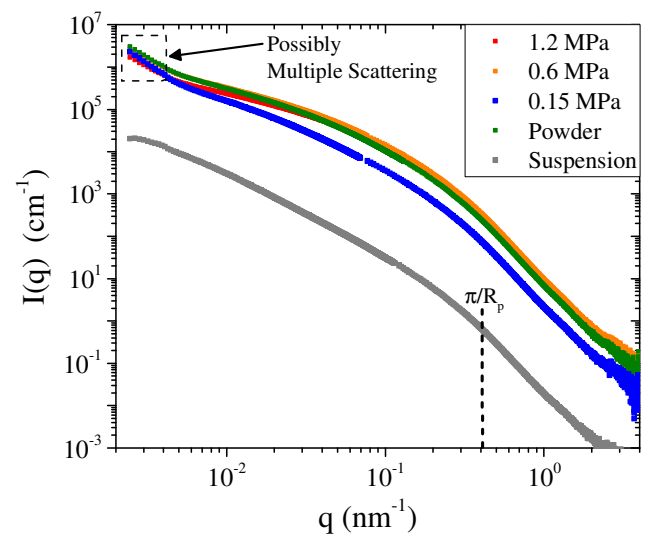

b)

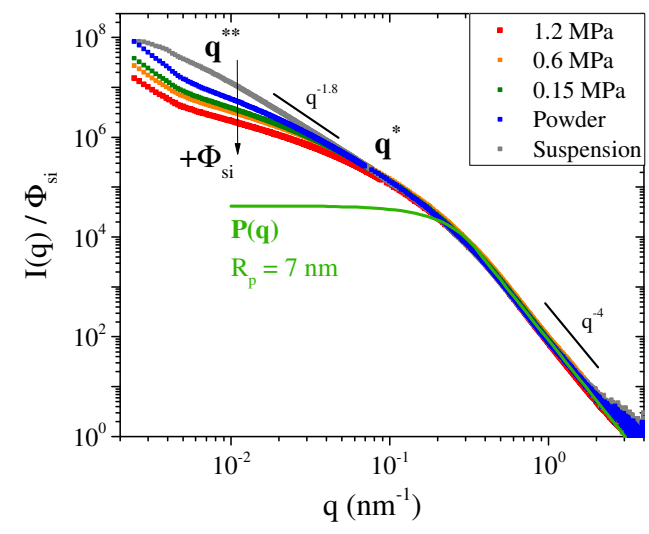

c)

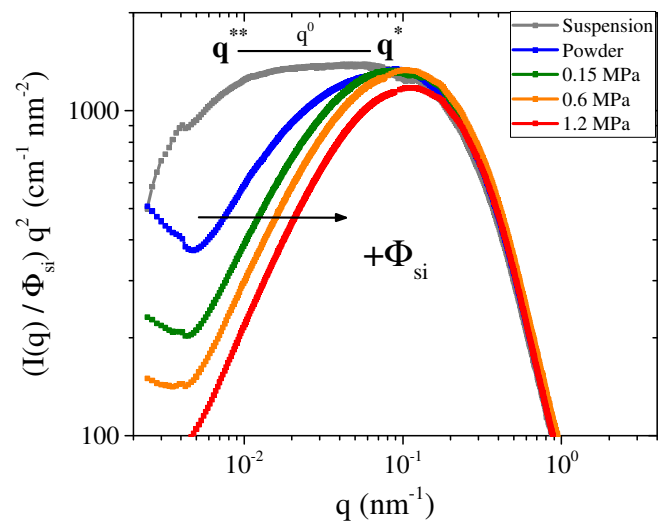

Figure 3: SAXS intensity measured from the fumed silica in various environment. a) Scattering intensity $I(q)$ as a function of the momentum transfer $q$ for fumed silica based samples (pellets pressed at 0.15 , o.6 and 1.2 MPa, corresponding unpressed powder and suspension in ethanol). Very high intensities (above $10^{6} \mathrm{~cm}^{-1}$ ) might be related with multiple scattering. b) Normalized scattering intensity $I(q) / \Phi_{s i}-$ The signals from the unpressed powder and the suspension (once corrected from the contrast) are shifted vertically to extract the corresponding $\Phi_{s i}$. The green solid line stands for a "Guinier-Porod" calculation with a particle radius $R_{p}=7 \mathrm{~nm}$ and a silicaair contrast $\Delta_{\text {silica-air }}=2.9410^{22} \mathrm{~cm}^{-4}$. c) $\log$-log Kratky representation $I(q) q^{2}=f(q)$ of the data presented in b). 
a)

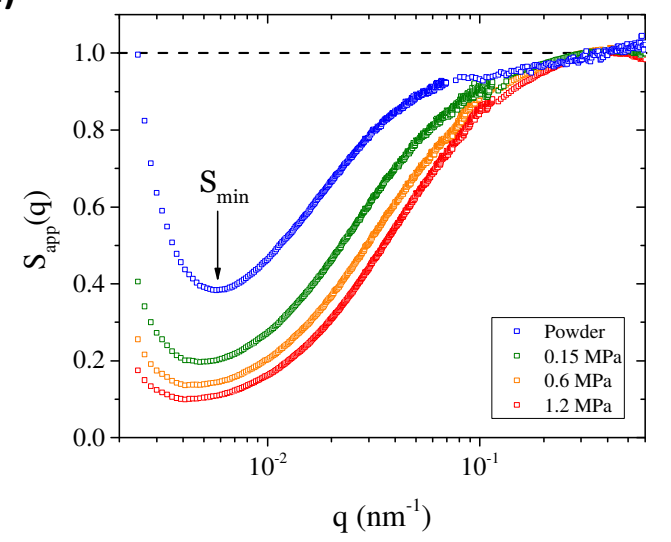

b)

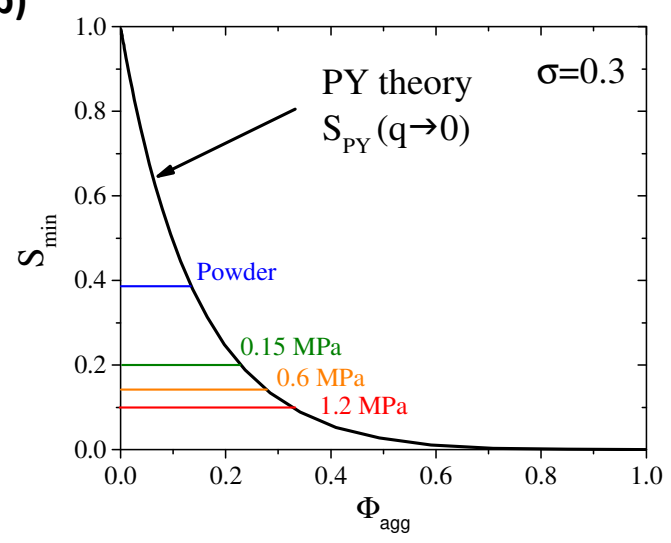

Figure 4: Analysis of the apparent structure factor calculated from SAXS experiments. a) Apparent structure factor obtained from $S_{a p p}(q)=$ $I(q) /\left(I_{0} P_{a g g l o}(q)\right)$ for the neat powder and the corresponding pellets compressed at 0.15, 0.6 and 1.2 MPa respectively. $S_{\min }$ values are reported in Table 1. b) Minimum value of the apparent structure factor $S_{\min }$ as a function of the volume fraction in silica aggregates $\Phi_{a g g}$. The solid line stands for the prediction calculated from the Percus-Yevick theory assuming a polydispersity of $\sigma=0.3$ (equation 3). 
a)

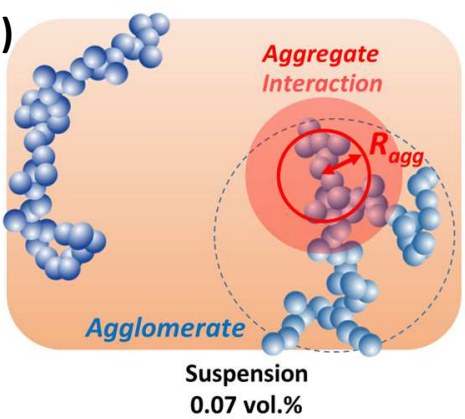

b)

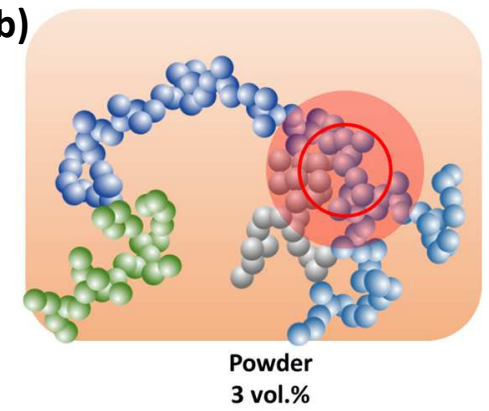

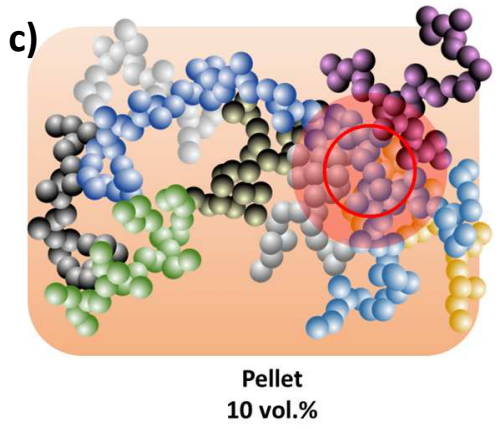

Figure 5: Schematic representation of the agglomerates and aggregates in the different samples. a) the ethanol suspension, b) the (uncompressed) powder and c) the compressed pellets. The red circle stands for the size of the aggregates and the arrow represents their radius. The shaded disk highlights the number of neighbor increasing with the volume fraction in silica, explaining the raise of the aggregates apparent fractal dimension estimated from the SAXS data analysis (its radius is set to $\left.2 R_{a g g}\right)$. 


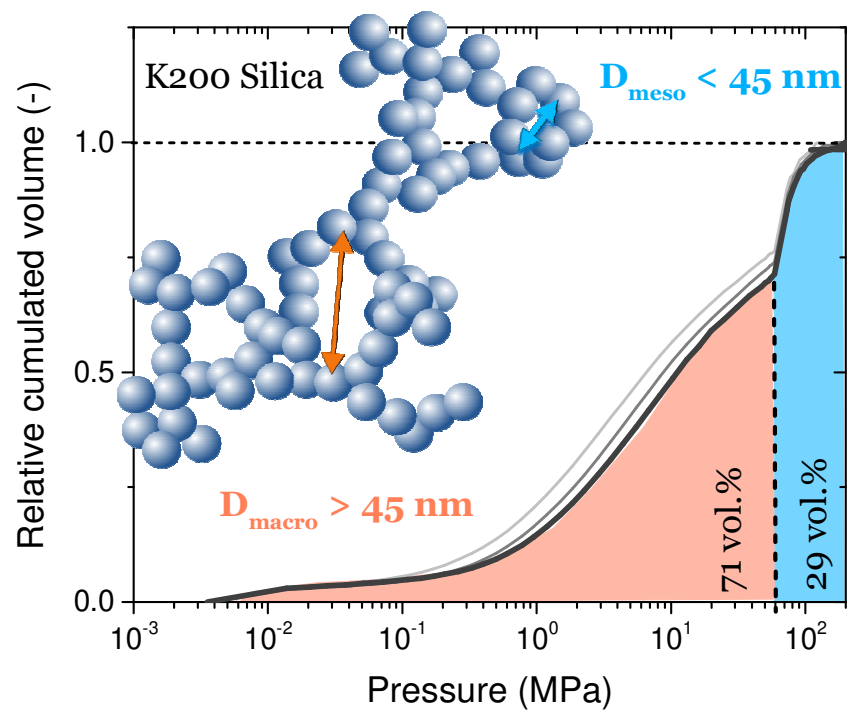

Figure 6: Mercury porosimetry experiment. Fraction of collapsed or intruded volume as a function of the mercury pressure. Each level of grey stands for an independent measurement giving an idea of the error bar. The inflexion point around $59 \mathrm{MPa}$ stands for the limit between the inter-aggregate ("macro") and intraaggregate ("meso") pores of radius $D_{\text {macro }}$ and $D_{\text {meso }}$ respectively. 


\section{References}

[1] G.P. Baeza, A.-C. Genix, C. Degrandcourt, L. Petitjean, J. Gummel, M. Couty, J. Oberdisse, Multiscale Filler Structure in Simplified Industrial Nanocomposite Silica/SBR Systems Studied by SAXS and TEM, Macromolecules 46(1) (2013) 317-329.

[2] G.P. Baeza, A.-C. Genix, C. Degrandcourt, J. Gummel, M. Couty, J. Oberdisse, Mechanism of aggregate formation in simplified industrial silica styrene-butadiene nanocomposites: effect of chain mass and grafting on rheology and structure, Soft Matter 10(35) (2014) 66866695 .

[3] D. Musino, A.-C. Genix, C. Fayolle, A.l. Papon, L. Guy, N. Meissner, R. Kozak, P. Weda, T. Bizien, T. Chaussé e, Synergistic Effect of Small Molecules on Large-Scale Structure of Simplified Industrial Nanocomposites, Macromolecules 50(13) (2017) 5138-5145.

[4] G.J. Schneider, V. Vollnhals, K. Brandt, S. Roth, D. Göritz, Correlation of mass fractal dimension and cluster size of silica in styrene butadiene rubber composites, J. Chem. Phys. 133(9) (2010) 094902.

[5] N. Suzuki, M. Ito, F. Yatsuyanagi, Effects of rubber/filler interactions on deformation behavior of silica filled SBR systems, Polymer 46(1) (2005) 193-201.

[6] I.A. Rahman, V. Padavettan, Synthesis of silica nanoparticles by sol-gel: size-dependent properties, surface modification, and applications in silica-polymer nanocomposites-a review, J. Nanomater. 2012 (2012) 8.

[7] L. Forny, I. Pezron, K. Saleh, P. Guigon, L. Komunjer, Storing water in powder form by self-assembling hydrophobic silica nanoparticles, Powder Technol. 171(1) (2007) 15-24. [8] S. Forrest, T. Witten Jr, Long-range correlations in smoke-particle aggregates, . Phys. A: Math. Gen. 12(5) (1979) L109.

[9] S.A. Johnson, P.J. Ollivier, T.E. Mallouk, Ordered mesoporous polymers of tunable pore size from colloidal silica templates, Science 283(5404) (1999) 963-965.

[10] G. Orts-Gil, K. Natte, D. Drescher, H. Bresch, A. Mantion, J. Kneipp, W. Österle, Characterisation of silica nanoparticles prior to in vitro studies: from primary particles to agglomerates, J. Nanopart. Res. 13(4) (2011) 1593-1604. 
[11] P.-J. De Temmerman, E. Van Doren, E. Verleysen, Y. Van der Stede, M.A.D. Francisco, J. Mast, Quantitative characterization of agglomerates and aggregates of pyrogenic and precipitated amorphous silica nanomaterials by transmission electron microscopy, J. Nanobiotechnology 10(1) (2012) 24.

[12] H. Singh, M. Geisler, F. Menzel, Experimental investigations into thermal transport phenomena in vacuum insulation panels (VIPs) using fumed silica cores, Energy Build. 107 (2015) 76-83.

[13] E.H. Kennard, Kinetic theory of gases, with an introduction to statistical mechanics, (1938).

[14] H. Gesser, P. Goswami, Aerogels and related porous materials, Chem. Rev. 89(4) (1989) 765-788.

[15] G. Reichenauer, U. Heinemann, H.-P. Ebert, Relationship between pore size and the gas pressure dependence of the gaseous thermal conductivity, Colloids Surf., A. 300(1-2) (2007) 204-210.

[16] M. Koebel, A. Rigacci, P. Achard, Aerogel-based thermal superinsulation: an overview, J. Sol-Gel Sci. Technol. 63(3) (2012) 315-339.

[17] J. Fricke, U. Heinemann, H. Ebert, Vacuum insulation panels-From research to market, Vacuum 82(7) (2008) 680-690.

[18] B. Yrieix, B. Morel, G. Foray, A. Bogner, Aerogel-based material that is super-insulating at atmospheric pressure, Google Patents, 2014.

[19] R.H. Nosrati, U. Berardi, Hygrothermal characteristics of aerogel-enhanced insulating materials under different humidity and temperature conditions, Energy Build. 158 (2018) 698-711.

[20] R. Baetens, B.P. Jelle, A. Gustavsen, Aerogel insulation for building applications: a stateof-the-art review, Energy Build. 43(4) (2011) 761-769.

[21] J. Fricke, U. Heinemann, H.P. Ebert, Vacuum insulation panels-From research to market, Vacuum 82(7) (2008) 680-690. 
[22] P. Johansson, C.-E. Hagentoft, A.S. Kalagasidis, Retrofitting of a listed brick and wood building using vacuum insulation panels on the exterior of the facade: Measurements and simulations, Energy Build. 73 (2014) 92-104.

[23] R. Baetens, B.P. Jelle, J.V. Thue, M.J. Tenpierik, S. Grynning, S. Uvsløkk, A. Gustavsen, Vacuum insulation panels for building applications: A review and beyond, Energy Build. 42(2) (2010) 147-172.

[24] F. Asdrubali, F. D'Alessandro, S. Schiavoni, A review of unconventional sustainable building insulation materials, Sustainable Mater. Technol. 4 (2015) 1-17.

[25] S. Schiavoni, F. Bianchi, F. Asdrubali, Insulation materials for the building sector: A review and comparative analysis, Renewable Sustainable Energy Rev. 62 (2016) 988-1011. [26] L. Aditya, T. Mahlia, B. Rismanchi, H. Ng, M. Hasan, H. Metselaar, O. Muraza, H. Aditiya, A review on insulation materials for energy conservation in buildings, Renewable Sustainable Energy Rev. 73 (2017) 1352-1365.

[27] S. Brunner, K.G. Wakili, T. Stahl, B. Binder, Vacuum insulation panels for building applications-Continuous challenges and developments, Energy Build. 85 (2014) 592-596. [28] P. Johansson, S. Geving, C.-E. Hagentoft, B.P. Jelle, E. Rognvik, A.S. Kalagasidis, B. Time, Interior insulation retrofit of a historical brick wall using vacuum insulation panels: Hygrothermal numerical simulations and laboratory investigations, Build. Environ. 79 (2014) $31-45$

[29] R. Caps, U. Heinemann, M. Ehrmanntraut, J. Fricke, Evacuated insulation panels filled with pyrogenic silica powders: properties and applications, High Temp. High Press. 33(2) (2001) $151-156$.

[30] M. Heine, S.E. Pratsinis, High concentration agglomerate dynamics at high temperatures, Langmuir 22(24) (2006) 10238-10245.

[31] D.W. Schaefer, A.J. Hurd, Growth and structure of combustion aerosols: fumed silica, Aerosol Sci. Technol. 12(4) (1990) 876-890.

[32] T.A. Witten, L.M. Sander, Diffusion-limited aggregation, Phys. Rev. B: Condens. Matter 27(9) (1983) 5686. 
[33] I. Rahman, P. Vejayakumaran, C. Sipaut, J. Ismail, M.A. Bakar, R. Adnan, C. Chee, An optimized sol-gel synthesis of stable primary equivalent silica particles, Colloids Surf., A. 294(1-3) (2007) 102-110.

[34] R. K. Iler, The chemistry of silica, Solubility, Polymerization, Colloid and Surface Properties and Biochemistry of Silica (1979).

[35] D. Quenard, Long-term performance of super insulating materials in building components \& systems-IEA-EBC Annex 65, Proceedings of International Conference CISBAT 2015 Future Buildings and Districts Sustainability from Nano to Urban Scale, LESO-PB, EPFL, 2015, pp. 125-130.

[36] M. Ramos, M. Gil, E. Schacht, G. Matthys, W. Mondelaers, M. Figueiredo, Physical and chemical characterisation of some silicas and silica derivatives, Powder Technol. 99(1) (1998) $79-85$

[37] J.W. Krumpfer, T. Schuster, M. Klapper, K. Müllen, Make it nano-Keep it nano, Nano Today 8(4) (2013) 417-438.

[38] www.oci.co.kr/product/product filedownload.asp?idx=1276.).

[39] P.A. Midgley, M. Weyland, 3D electron microscopy in the physical sciences: the development of Z-contrast and EFTEM tomography, Ultramicroscopy 96(3) (2003) 413-431. [40] M. Weyland, P.A. Midgley, Electron tomography, Materials Today 7(12) (2004) 32-40. [41] S. Carenco, S. Moldovan, L. Roiban, I. Florea, D. Portehault, K. Vallé, P. Belleville, C. Boissière, L. Rozes, N. Mézailles, The core contribution of transmission electron microscopy to functional nanomaterials engineering, Nanoscale 8(3) (2016) 1260-1279.

[42] O. Ersen, I. Florea, C. Hirlimann, C. Pham-Huu, Exploring nanomaterials with 3D electron microscopy, Materials Today 18(7) (2015) 395-408.

[43] L. Roiban, G. Foray, Q. Rong, A. Perret, D. Ihiawakrim, K. Masenelli-Varlot, E. Maire, B. Yrieix, Advanced three dimensional characterization of silica-based ultraporous materials, RSC Advances 6(13) (2016) 10625-10632.

[44] J.R. Kremer, D.N. Mastronarde, J.R. McIntosh, Computer Visualization of ThreeDimensional Image Data Using IMOD, J. Struct. Biol. 116(1) (1996) 71-76. 
[45] C. MessaoudiI, T. Boudier, C.O.S. Sorzano, S. Marco, TomoJ: tomography software for three-dimensional reconstruction in transmission electron microscopy, BMC Bioinf. 8(1) (2007) 288.

[46] S. Bolte, F. Cordelieres, A guided tour into subcellular colocalization analysis in light microscopy, J. Microsc. 224(3) (2006) 213-232.

[47] D. Tschumperle, R. Deriche, Vector-valued image regularization with PDEs: A common framework for different applications, IEEE transactions on pattern analysis and machine intelligence 27(4) (2005) 506-517.

[48] A. Fedorov, R. Beichel, J. Kalpathy-Cramer, J. Finet, J.-C. Fillion-Robin, S. Pujol, C. Bauer, D. Jennings, F. Fennessy, M. Sonka, J. Buatti, S. Aylward, J.V. Miller, S. Pieper, R. Kikinis, 3D Slicer as an image computing platform for the Quantitative Imaging Network, Magn. Reson. Imaging 30(9) (2012) 1323-1341.

[49] D. Boldridge, Morphological characterization of fumed silica aggregates, Aerosol Sci. Technol. 44(3) (2010) 182-186.

[50] P. Lindner, T. Zemb, Neutrons, X-rays and Light: Scattering Methods Applied to Soft Condensed Matter, in: North-Holland (Ed.) 2002.

[51] B. Hammouda, A new Guinier-Porod model, J. Appl. Crystallogr. 43(4) (2010) 716-719. [52] A.-C. Genix, J. Oberdisse, Determination of the local density of polydisperse nanoparticle assemblies, Soft Matter 13(44) (2017) 8144-8155.

[53] G.P. Baeza, A.-C. Genix, N. Paupy-Peyronnet, C. Degrandcourt, M. Couty, J. Oberdisse, Revealing nanocomposite filler structures by swelling and small-angle X-ray scattering, Faraday Discuss. 186(0) (2016) 295-309.

[54] G.P. Baeza, A.-C. Genix, C. Degrandcourt, L. Petitjean, J. Gummel, R. Schweins, M. Couty, J. Oberdisse, Effect of Grafting on Rheology and Structure of a Simplified Industrial Nanocomposite Silica/SBR, Macromolecules 46(16) (2013) 6621-6633.

[55] F. Saint-Michel, F. Pignon, A. Magnin, Fractal behavior and scaling law of hydrophobic silica in polyol, J. Colloid Interface Sci. 267(2) (2003) 314-319. 
[56] G.J. Schneider, Correlation of mass fractal dimension and asymmetry, J. Chem. Phys. 130(23) (2009) 234912.

[57] H. Montes, A. Papon, F. Lequeux, L. Guy, Particles in model filled rubber: dispersion and mechanical properties, Eur. Phys. J. E 31(3) (2010) 263-268.

[58] D. Musino, A.-C. Genix, T. Chausse' e, L. Guy, N. Meissner, R. Kozak, T. Bizien, J. Oberdisse, Aggregate Formation of Surface-Modified Nanoparticles in Solvents and Polymer Nanocomposites, Langmuir 34(9) (2018) 3010-3020.

[59] J. Oberdisse, Aggregation of colloidal nanoparticles in polymer matrices, Soft Matter 2(1) (2006) 29-36.

[6o] J.K. Percus, G.J. Yevick, Analysis of classical statistical mechanics by means of collective coordinates, Phys. Rev. 110(1) (1958) 1.

[61] N. Ashcroft, D.C. Langreth, Structure of binary liquid mixtures. I, Phys. Rev. 156(3) (1967) 685.

[62] S. Oh, B. Kim, H. Kim, Comparison of nanoparticle exposures between fumed and solgel nano-silica manufacturing facilities, Ind. health 52(3) (2014) 190-198.

[63] C. Oh, C.M. Sorensen, The Effect of Overlap between Monomers on the Determination of Fractal Cluster Morphology, J. Colloid Interface Sci. 193(1) (1997) 17-25.

[64] R. Wengeler, H. Nirschl, Turbulent hydrodynamic stress induced dispersion and fragmentation of nanoscale agglomerates, J. Colloid Interface Sci. 306(2) (2007) 262-273. [65] H.K. Kammler, G. Beaucage, R. Mueller, S.E. Pratsinis, Structure of flame-made silica nanoparticles by ultra-small-angle X-ray scattering, Langmuir 20(5) (2004) 1915-1921. [66] E. Garboczi, K. Snyder, J. Douglas, M. Thorpe, Geometrical percolation threshold of overlapping ellipsoids, Phys Rev. E 52(1) (1995) 819.

[67] S. Meille, E.J. Garboczi, Linear elastic properties of 2D and 3D models of porous materials made from elongated objects, Modell. Simul. Mater. Sci. Eng. 9(5) (2001) 371. [68] J. Sanahuja, L. Dormieux, S. Meille, C. Hellmich, A. Fritsch, Micromechanical explanation of elasticity and strength of gypsum: from elongated anisotropic crystals to isotropic porous polycrystals, J. Eng. Mech. 136(2) (2009) 239-253. 
[69] D.M. Smith, G.P. Johnston, A.J. Hurd, Structural studies of vapor-phase aggregates via mercury porosimetry, J. Colloid Interface Sci. 135(1) (1990) 227-237.

[70] K. Xu, J.-f. Daian, D. Quenard, Multiscale structures to describe porous media part I: theoretical background and invasion by fluids, Transp. Porous Media 26(1) (1997) 51-73. [71] G. Johnston, D. Smith, I. Melendez, A. Hurd, Compression effects in mercury porosimetry, Powder Technol. 61(3) (1990) 289-294.

[72] D. Julve, J. Ramos, J. Pérez, M. Menéndez, Analysis of mercury porosimetry curves of precipitated silica, as an example of compressible porous solids, J. Non-Cryst. Solids 357(4) (2011) 1319-1327. 


\section{USAXS}

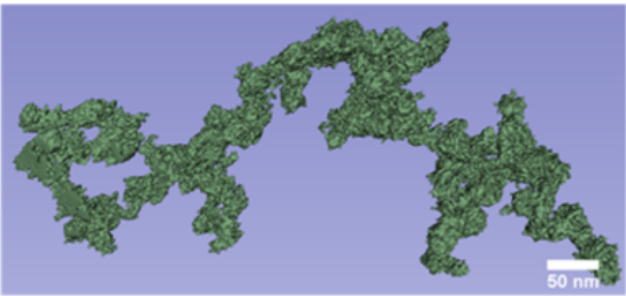

POROO

UNRAVELLING THE FUMED SILICA MULTISCALE-STRUCTURE

\section{TOMO}

TEM 